\title{
Improving Pseudo-Relevance Feedback in Web Information Retrieval Using Web Page Segmentation
}

\author{
Shipeng $\mathrm{Yu}^{1^{*}}$ Deng $\mathrm{Cai}^{2^{*}} \mathrm{Ji-Rong} \mathrm{Wen}{ }^{*}$ and Wei-Ying Ma*
}

\author{
Microsoft Research Asia \\ Beijing, China
}

\{jrwen, wyma\}@microsoft.com

\author{
${ }^{1}$ Peking University \\ Beijing, China \\ ysp@is.pku.edu.cn
}

\author{
${ }^{2} T$ singhua University \\ Beijing, China \\ caideng00@mails.tsinghua.edu.cn
}

\begin{abstract}
In contrast to traditional document retrieval, a web page as a whole is not a good information unit to search because it often contains multiple topics and a lot of irrelevant information from navigation, decoration, and interaction part of the page. In this paper, we propose a VIsion-based Page Segmentation (VIPS) algorithm to detect the semantic content structure in a web page. Compared with simple DOM based segmentation method, our page segmentation scheme utilizes useful visual cues to obtain a better partition of a page at the semantic level. By using our VIPS algorithm to assist the selection of query expansion terms in pseudo-relevance feedback in web information retrieval, we achieve $27 \%$ performance improvement on Web Track dataset.
\end{abstract}

\section{Categories and Subject Descriptors}

H.3.3 [Information Storage and Retrieval]: Information Search and Retrieval - Relevance feedback; H.5.4 [Information Interfaces and Presentation]: Hypertext/Hypermedia

\section{General Terms}

Algorithms, Performance, Human Factors

\section{Keywords}

Web information retrieval, page segmentation, query expansion, relevance feedback

\section{INTRODUCTION}

To cope with the information explosion of the Web, effective and efficient information retrieval has been the most challenging problem for a search engine. Pseudo-relevance feedback, also known as local feedback or blind feedback, is a technique commonly used to improve retrieval performance $[3,9]$. Its basic idea is to extract expansion terms from the top-ranked documents to formulate a new query for a second round retrieval. Through a query expansion, some relevant documents missed in the initial round can then be retrieved to improve the overall performance. Clearly, the effect of this method strongly relies on the quality of selected expansion terms. If the words added to the original query are unrelated to the topic, the quality of the retrieval is likely to be degraded. Since the Web is a highly volatile and heterogeneous

Copyright is held by the author/owner(s). WWW 2003, May 20-24, 2003, Budapest, Hungary. ACM xxx. information source and contains a lot of low-quality documents [1], such a naive pseudo-relevance feedback is not capable of producing a satisfactory result.

There are many reasons why simple pseudo-relevance feedback does not work. First, web pages usually do not contain pure content. A web page typically contains various types of materials that are not related to the topic of the web-page, such as:

$\diamond \quad$ Navigation - intra and inter hyperlinks to guide the user to different part of a web page.

$\diamond$ Decoration - pictures, animations, logos and so forth for attraction or advertisement purposes.

$\diamond \quad$ Interaction - forms to collect user information or provide searching services.

$\diamond \quad$ Other special words or paragraphs such as copyrights and contact information.

In terms of query matching and term weighting, all of the above stuffs are considered noises and harmful to retrieval performance. Secondly, a web page usually contains multiple topics, for example, a news page containing many different comments on a particular event or politician, or a conference web page containing sponsors from different companies and organizations. Although traditional documents also often have multiple topics, they are less diverse so that the impact on retrieval performance is smaller.

For pseudo-relevance feedback, the quality of expansion terms is heavily affected by the top-ranked documents. Noise and multitopics are two major negative factors for expansion term selection. For instance, if an advertisement is embedded in a top-ranked web page at the first round, then some terms from the advertisement may be selected as expansion terms. Once these terms are used to expand the query for the second round retrieval, irrelevant web pages containing these advertisement terms could be ranked highly. Similarly, for a web page containing multiple topics, the selected terms are also subject to this uncertainty which may decrease the retrieval performance. Therefore, it is necessary to segment a web page into semantically related units so that noisy information can be filtered out and multiple topics can be distinguished.

The structure of a HTML page can be represented as a tag tree by DOM (Document Object Model, see http://www.w3.org/DOM/). DOM-based segmentation approaches are widely used these years for record boundary discovery $[4,10]$, topic distillation [6] and adaptive content delivery $[5,7,12]$. Useful tags that may 
represent a block in a page include $P$ (for paragraph), TABLE (for table), $U L$ (for list), H1 H6 (for heading), etc. To the best of our knowledge, few works are done on applying DOM-based page segmentation methods to improve web information retrieval. Some preliminary studies are performed in $[8,13]$ but the results are not encouraging. DOM in general provides a useful structure for a web page. But tags such as TABLE and $P$ are used not only for content organization, but also for layout presentation. In many cases, DOM tends to reveal presentation structure other than content structure [11], and is often not accurate enough to discriminate different semantic blocks in a web page.

To segment a web page into semantically related units, the visual presentation of the page contains a lot of useful cues. Generally, a web page designer would organize the content of a web page to make it easy for reading. Thus, semantically related content is usually grouped together and the entire page is divided into regions for different contents using explicit or implicit visual separators such as lines, blank areas, images, font sizes, colors, etc [17]. Our goal is to derive this content structure from the visual presentation of a web page. In this paper, we propose VIPS (VIsion-based Page Segmentation) algorithm to segment a web page. The algorithm makes full use of the layout features of the page and tries to partition the page at the semantic level. Each node in the extracted content structure will correspond to a block of coherent content in the original page.

Compared with DOM based methods, the segments obtained by VIPS are much more semantically aggregated. Noisy information, such as navigation, advertisement, and decoration can be easily removed because they are often the blocks in some fixed region of a page. Content with different topics is distinguished as separate blocks. With the assumption that terms in different segments are not correlated to a common topic, the expansion term selection is constrained within a few segments instead of the whole web page. By combining VIPS algorithm with the pseudo-relevance feedback method, we propose a novel segment-based pseudorelevance feedback method for web information retrieval. The experimental results in Section 4 prove that our proposed method can significantly improve the retrieval performance, both in terms of precision and recall.

The rest of the paper is organized as the following. The details of the VIPS algorithm are introduced in Section 2. In Section 3, we illustrate how to utilize web page segmentation to improve pseudo-relevance feedback. The experimental results are reported in Section 4. Section 5 summarizes our contributions and concludes the paper.

\section{THE VIPS ALGORITHM}

People view a web page through a web browser and get a 2-D presentation which provides many visual cues to help distinguish different parts of the page. Examples of these cues include lines, blanks, images, different font sizes, and different colors, etc. For the purpose of easy browsing and understanding, a closely packed region within a web page is usually about a single topic. This observation motivates us to segment a web page from its visual presentation

In [17] and [7], some visual cues are used in DOM analysis. They try to identify the logical relationships within a web page based on visual layout information, but these approaches rely too much on the DOM structure. Gu [11] tries to construct a web content structure by breaking out the DOM tree and comparing similarity among all the basic DOM nodes. Since a normal web page may have hundreds of basic elements, the algorithm is time-consuming and inflexible, not suitable to deal with a large amount of web pages.

We propose a vision-based web page segmentation algorithm by combining the DOM structure and visual cues. After the segmentation process, a tree-like content structure of the page based on its visual presentation will be constructed. Take the web page in Figure 1 as an example (this web page can be accessed at http://catless.ncl.ac.uk/Risks/22.06.html with a little modification for simplicity). The tree on the left side is the DOM structure of the page. Since tags are distributed within the $B O D Y$ tag without any hierarchies, it is hard to directly extract segments from the DOM structure that correspond to a visual block and contain a unit of information in the page. However from the presentation style, we can easily distinguish each part from the others and recognize the topic of each part in the page, as illustrated on the right side of the page.

\subsection{Vision-Based Content Structure for Web Pages}

The vision-based content structure of a web page is a tree-like structure. Each node of the tree represents a region in the web page, which we call a visual block or $v b$ for abbreviation. If a node has children in the structure, visual blocks of the children are contained within that of the node and form a partition of it. As shown in right side of Figure 1, the visual blocks $V B 1-2-1$ and $V B 1-2-2$ are children of $V B 1-2$ and act as a partition in the page.

For each node, the Degree of Coherence $(D o C)$ is defined to show how coherent it is. The value of $D o C$ usually ranges from 0 to 1 . We can pre-define the Permitted Degree of Coherence (PDoC) to achieve different granularities of page segmentation for different applications. The less the $P D o C$ is, the coarser the content structure would be. For example, in Figure 1, the visual block VB1-2-2-2-2 may not be further partitioned with an appropriate $P D o C$.

The vision-based content structure is more likely to provide a semantic partitioning of the page. Every node of the structure, especially the leaf node, is more likely to convey a certain semantic meaning and help to build a higher semantic via the hierarchy. For instance, in Figure 1 we can say that VB1-2-1 denotes the title of the page, $V B 1-2-2-2-1$ shows table of contents and $V B 1-2-2-2-2$ tells a whole story. 


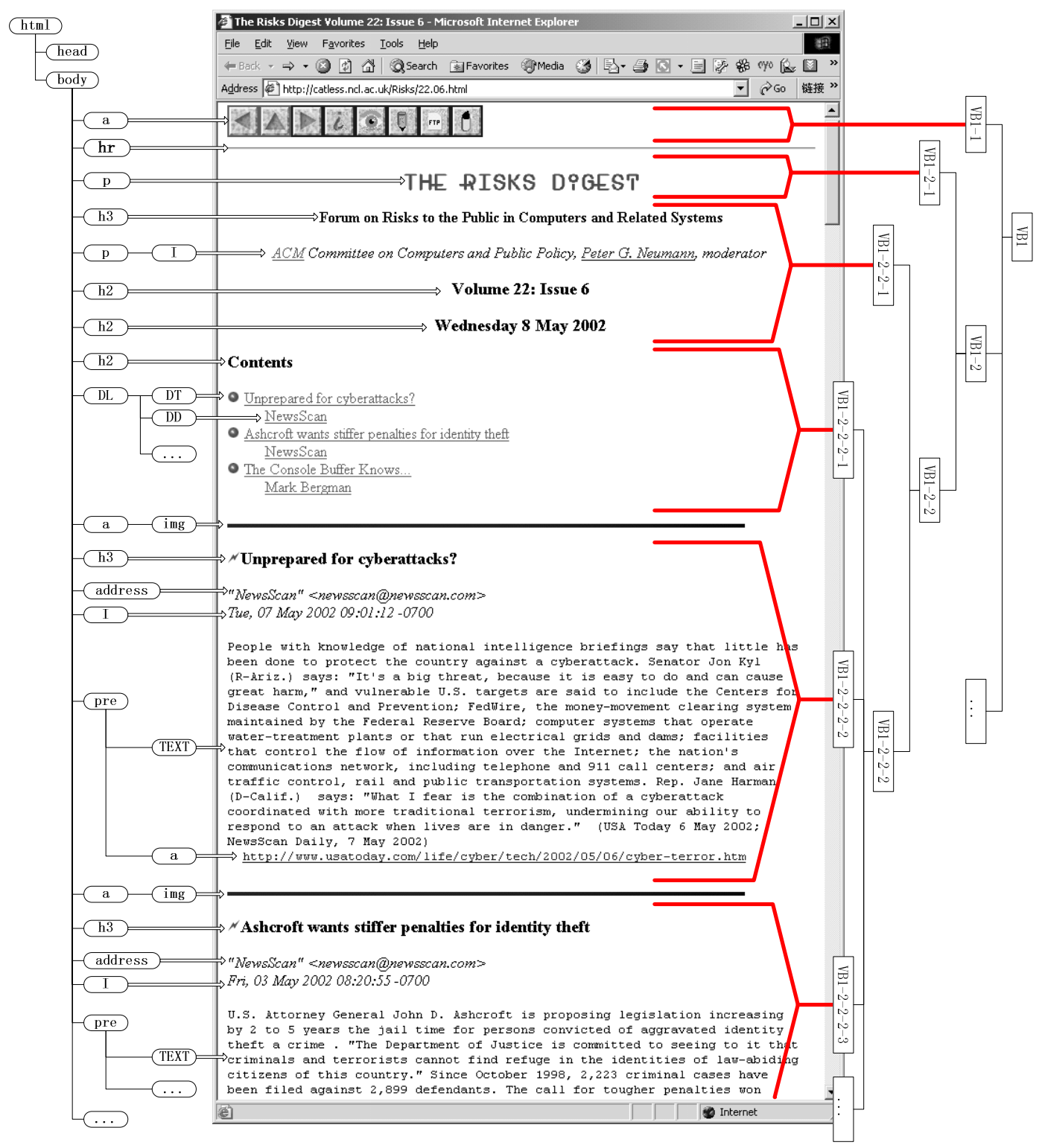

Figure 1. A sample web page with its DOM structure on the left and our extracted vision-based content structure on the right

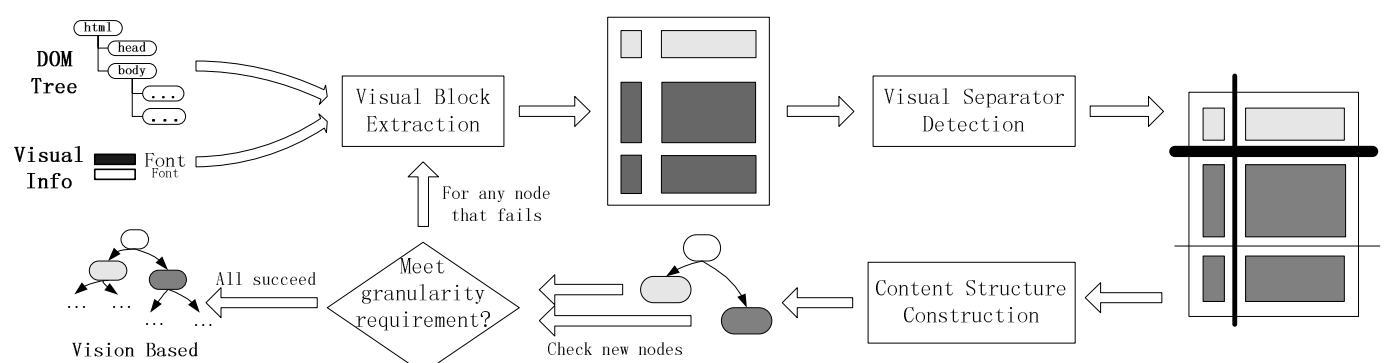

Figure 2. Flowchart of the VIPS algorithm 


\subsection{Vision-based Page Segmentation Algorithm}

To obtain the vision-based content structure for a web page, we introduce the VIPS (Vision-based Page Segmentation) algorithm which makes use of DOM structure as well as visual cues. The flowchart of the segmentation process is illustrated in Figure 2.

First, by calling the analyzer embedded in the web browser, we obtain the DOM structure and visual information such as position, color, font size, font weight, etc. All the visual information comes from HTML elements and attributes. From the subtree within $B O D Y$ in the DOM structure, we start the following iterative process to build the content structure.

\section{Step 1: Visual Block Extraction}

In this phase, we aim at finding all appropriate visual blocks contained in the current subtree. Normally, every node inside a current node can represent a visual block, like all the children of $B O D Y$ in Figure 1. However, some "huge" nodes such as TABLE and $P$ may act only for organization purpose and are not appropriate to represent a single visual block. Therefore, in these cases we should further divide the current node and replace it by its children. This process is iterated until all appropriate nodes are found to represent the visual blocks in the web page.

At the end of this step, for each node that represents a visual block, its $D o C$ value is set according to its intra visual difference. Some heuristic rules are used to determine whether a DOM node should be replaced as the following:

$\diamond \quad$ Tag cue: Tags such as $H R$ are often used to separate different topics from visual perspective. Therefore we prefer to divide a DOM node if it contains these tags.

$\diamond \quad$ Color cue: We divide a DOM node if its background color is different from one of its children's.

$\diamond \quad$ Text cue: If most of the children of a DOM node are Text nodes (i.e., no tags surround them), we do not divide it.

$\diamond \quad$ Size cue: We prefer to divide a DOM node if the standard deviation of size of its children is larger than a threshold.

In Figure 1, the BODY tag will be divided according to Tag cue and Size cue. Among all of its children, node PRE will not be divided according to Text cue.

\section{Step 2: Visual Separator Detection}

When all blocks are extracted, they are put into a pool for separator detection. We define visual separators as horizontal or vertical lines in a web page that visually cross with no blocks in the pool. We set appropriate weight to each separator according to the following patterns and select those with highest weight as the actual separators.

$\diamond \quad$ Distance pattern: The more the distance between blocks on different side of the separator, the higher the weight.

$\diamond \quad$ Tag pattern: If a visual separator is at the same position as some tags such as $H R$, its weight is made higher.

$\diamond \quad$ Font pattern: If differences of font properties such as font size and font weight are more clearly on two sides of the separator, the weight will be higher. $\diamond \quad$ Color pattern: If background colors are different on two sides of the separator, the weight will be higher.

In Figure 1, because all the visual blocks are parallel under the DOM node $B O D Y$, we get many horizontal separators. In the first iteration, the separator with a $H R$ in its position gets the maximal weight. In the second iteration, the separator below "THE RESKS DYGEST" is selected as the actual separator according to Font pattern.

\section{Step 3: Content Structure Construction}

When the actual separators are detected, visual blocks on the same sides of all the separators are merged and represented as a node in the content structure. The $D o C$ of each node is also defined through similar methods as described in Step 1. After that, each node is checked whether it meets the granularity requirement. For every node that fails, we go to Step 1 on Visual Block Extraction phase again to further construct the sub content structure within the node. If all the nodes meet the requirement, the iterative process is then stopped and the vision-based content structure for the whole page is obtained. The common requirement for $D o C$ is that $\mathrm{DoC}>\mathrm{PD} o \mathrm{C}$, if $P D o C$ is pre-defined.

Go back to the example in Figure 1. In the first iteration, the actual separator found in Step 2 results two nodes $V B 1-1$ and $V B 1-2$. $V B 1-1$ meets the $D o C$ requirement and needs no further processing, while $V B 1-2$ goes through another iteration and gets several children. After several iterations, the final vision-based content structure of the page is constructed.

The proposed VIPS algorithm takes advantage of visual cues to obtain the vision-based content structure of a web page. The algorithm successfully bridges the gap between the DOM structure and the semantic structure. The page is partitioned based on visual separators and structured as a hierarchy closely related to how a user will browse the page. Content related parts could be grouped together even if they are in different branches of the DOM tree.

VIPS is also very efficient. Since we trace down the DOM structure for visual block extraction and do not analyze every basic DOM node, the algorithm is totally top-down. Furthermore, the $P D o C$ can be pre-defined, which brings significant flexibility to segmentation and greatly improve the performance.

\section{APPLYING VIPS ON PSEUDO- RELEVANCE FEEDBACK}

Since our VIPS algorithm can group semantically related content into a single segment, the term correlations within a segment will be much higher than those within other range of a whole web page. With the improved term correlations, high-quality expansion terms can be extracted from segments and then used to improve information retrieval performance. Therefore, we combine VIPS with the pseudo-relevance feedback algorithm according to the following steps:

\section{Step 1: Initial Retrieval}

An initial list of ranked web pages is obtained by using any traditional information retrieval methods.

\section{Step 2: Page Segmentation}

In this step, VIPS algorithm is applied to divide retrieved web pages into segments. After the vision-based content structure is 
obtained, all the leaf nodes are extracted as segments. Since it is very expensive to process all retrieved web pages, we only select a few (e.g. 80) top pages for segmentation. The candidate segment set is made up of these resulting segments.

\section{Step 3: Segment Selection}

This step aims to choose most relevant segments from the candidate segment set. Some ranking methods (such as BM2500 [16]) are used to sort the candidate segments and the top (e.g. 20) segments are selected for expansion term selection in the next step.

\section{Step 4: Expansion Term Selection}

We use an approach similar to the traditional pseudo-relevance feedback algorithm to select expansion terms. The difference is that expansion terms are selected from the selected segments instead of from the whole web pages. All terms except the original query terms in the selected segments are weighted according to the following term selection value TSV:

$$
T S V=w^{(1)} * r / R
$$

where $w^{(1)}$ is the Robertson/Sparck Jones weight [15]; $R$ is the number of selected segments; and $r$ is the number of segments which contain this term. In our experiments, the top 10 terms are selected to expand the original query.

\section{Step 5: Final Retrieval}

The term weights for the expanded query are set as the following:

$\diamond \quad$ For original terms, new weight is $t f * 2$ where $t f$ is its term frequency in the query;

$\diamond$ For expansion terms, new weight is $1-(n-1) / 10$ if the current term ranks $n$th in TSV rank. Note that we assume a total of 10 terms are selected to expand the query.

The expanded query is used to retrieve the data set again for the final results.

\section{EVALUATION}

This section provides empirical evidences on how web page segmentation can be used to greatly improve the performance of pseudo-relevance feedback. The following page segmentation methods are evaluated in this paper.

$\diamond \quad$ Our Vision-based approach (denoted as VIPS): The PDoC is set to 0.6 . To reduce the effect of tiny blocks, blocks less than 10 words are removed. The top 80 pages returned by the initial retrieval phase are segmented to form the candidate segment set.

$\diamond \quad$ Simple DOM-based approach (denoted as DOMPS): We iterate the DOM tree for some structural tags such as TITLE, $P, T A B L E, U L$ and $H 1 \sim H 6$. If there are no more structural tags within the current structural tag, a block is constructed and identified by this tag. Free text between two tags is also treated as a special block. Similar to VIPS, tiny blocks less than 10 words are also removed, and the candidate segments are chosen from the top 80 pages returned by the initial retrieval phase.

$\diamond \quad$ Traditional full document approach (denoted as FULLDOC): To evaluate how much performance gain we could achieve using page segmentation for expansion term selection, the traditional pseudo-relevance feedback based on the whole web page is implemented for a comparison purpose.

\subsection{Test Data and Configuration}

We choose Okapi [14] as the retrieval system and WT10g [2] in TREC-9 and TREC 2001 Web Tracks as the data set. WT10g contains 1.69 million pages and amounts to about 10G. We use the 50 queries from TREC 2001 Web Track as the query set and only the TOPIC field for retrieval. We use Okapi's BM2500 [16] as the weight function and set $k 1=1.2, k 3=1000, b=0.75$, and $a v d l=61200$.

In our experiments, we do not use any stemming method. A word list containing 222 words [16] is used to filter out stop words. We only use single word and do not consider phrase information.

\subsection{Experimental Results}

To show the effect of segment selection, we alter the number of segments from 3 to 60 in the experiments. Table 1 and Figure 3 illustrate the comparison of performance. In Table 1, for each segmentation algorithm, the value in bold is the best performance achieved. In Figure 3, the horizontal axis is the number of documents or blocks, and the vertical axis shows the average precision. The baseline is $16.55 \%$ and is shown with a dotted line. Results from different web page segmentation algorithms are shown respectively.

As can be seen, the average retrieval precision can be improved after partitioning pages into blocks, no matter which segmentation algorithm is used. In the case of FULLDOC, the maximal average precision is $19.10 \%$ when the top 10 documents are used to expand the query. DOMPS obtains $19.67 \%$ when the top 50 blocks are used, a little better than FULLDOC. VIPS gets the best result $20.98 \%$ when the top 20 blocks are used and achieves $26.77 \%$ improvement.

Table 1. Performance comparison of query expansion using different page segmentation methods

\begin{tabular}{|c|c|c|c|c|}
\hline $\begin{array}{l}\text { Number of } \\
\text { Segments }\end{array}$ & $\begin{array}{c}\text { Baseline } \\
(\%)\end{array}$ & $\begin{array}{c}\text { FULLDOC } \\
(\%)\end{array}$ & $\begin{array}{c}\text { DOMPS } \\
(\%)\end{array}$ & $\begin{array}{l}\text { VIPS } \\
(\%)\end{array}$ \\
\hline 3 & \multirow{8}{*}{16.55} & $\begin{array}{c}17.56 \\
(+6.10)\end{array}$ & $\begin{array}{c}17.94 \\
(+8.40)\end{array}$ & $\begin{array}{c}18.01 \\
(+8.82)\end{array}$ \\
\hline 5 & & $\begin{array}{c}17.46 \\
(+5.50)\end{array}$ & $\begin{array}{c}18.15 \\
(+9.67)\end{array}$ & $\begin{array}{c}19.39 \\
(+17.16)\end{array}$ \\
\hline 10 & & $\begin{array}{c}19.10 \\
(+15.41)\end{array}$ & $\begin{array}{c}18.05 \\
(+9.06)\end{array}$ & $\begin{array}{c}19.92 \\
(+20.36)\end{array}$ \\
\hline 20 & & $\begin{array}{c}17.89 \\
(+8.10)\end{array}$ & $\begin{array}{c}19.24 \\
(+16.25)\end{array}$ & $\begin{array}{c}20.98 \\
(+26.77)\end{array}$ \\
\hline 30 & & $\begin{array}{c}17.40 \\
(+5.14)\end{array}$ & $\begin{array}{c}19.32 \\
(+16.74)\end{array}$ & $\begin{array}{c}19.68 \\
(+18.91)\end{array}$ \\
\hline 40 & & $\begin{array}{l}15.50 \\
(-6.34)\end{array}$ & $\begin{array}{c}19.57 \\
(+18.25)\end{array}$ & $\begin{array}{c}17.24 \\
(+4.17)\end{array}$ \\
\hline 50 & & $\begin{array}{c}13.82 \\
(-16.50)\end{array}$ & $\begin{array}{c}19.67 \\
(+18.85)\end{array}$ & $\begin{array}{c}16.63 \\
(+0.48)\end{array}$ \\
\hline 60 & & $\begin{array}{c}14.40 \\
(-12.99)\end{array}$ & $\begin{array}{c}18.58 \\
(+12.27)\end{array}$ & $\begin{array}{c}16.37 \\
(-1.09)\end{array}$ \\
\hline
\end{tabular}




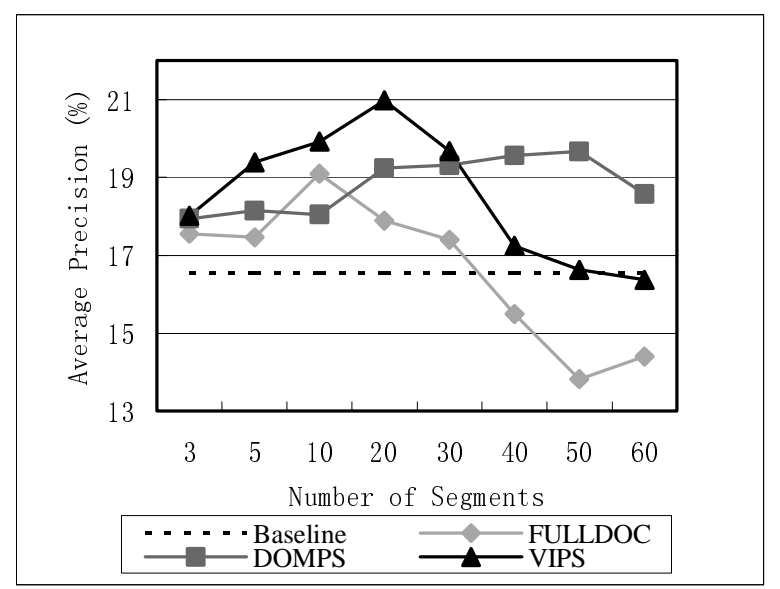

Figure 3. Performance comparison of query expansion using different page segmentation methods

Document based query expansion FULLDOC uses all the terms within the top documents for expansion. Since the baseline is very low, many of top ranked documents are actually irrelevant and there are many terms coming from irrelevant topics. These cause the retrieval performance relatively low although better than the baseline. For the same reasons, the average precision drops quickly when more documents are used to select expansion terms. It becomes lower than the baseline after 30 .

DOM based approach DOMPS does not obtain a significant improvement compared to FULLDOC, partly because the segmentation is too detailed. The average length of segments in DOM based approach is about 540 in byte. The segments are usually too short to cover complete information about a single semantic. In many cases, good expansion terms are within the previous or proceeding blocks, but are missed because those blocks are not ranked high enough to be selected in pseudorelevance feedback.

Compared with DOMPS, our VIPS algorithm considers more visual information and is more likely to obtain a semantic partition of a web page. Therefore, more good expansion terms can be extracted and better performance can be achieved. As can be seen from Figure 3, VIPS performs the best when less than 20 blocks are used in query expansion. The reason is that the top blocks are usually most relevant and thus contain very good expansion terms. After 20 blocks, more noises will be introduced and the results drop down quickly. Some other reasons for the quick drop lie in that many pages in our experiments are badly presented and that the block lengths vary remarkably.

From Figure 3 we can also see different performance peaks for the three approaches, which reflect different granularities of page segmentation. FULLDOC takes whole document for expansion term selection and has coarsest granularity, therefore arrives at its peak when only 10 documents are selected. DOMPS is the most detailed approach and gets the best performance when 50 blocks are used. VIPS gets its peak between the former two.

Table 2 provides the comparison of every recall level using the best result of each approach listed in Table 1. The VIPS approach performs the best in most recall levels.
Table 2. Comparison of precision at all recall levels using best results of different page segmentation methods

\begin{tabular}{|c|c|c|c|c|}
\hline $\begin{array}{c}\text { Recall } \\
(\%)\end{array}$ & $\begin{array}{c}\text { Baseline } \\
(\%)\end{array}$ & $\begin{array}{c}\text { FULLDOC } \\
(\%)\end{array}$ & $\begin{array}{c}\text { DOMPS } \\
(\%)\end{array}$ & $\begin{array}{l}\text { VIPS } \\
(\%)\end{array}$ \\
\hline 0 & 58.55 & $\begin{array}{c}62.04 \\
(+5.96)\end{array}$ & $\begin{array}{c}58.87 \\
(+0.54)\end{array}$ & $\begin{array}{c}59.31 \\
(+1.30)\end{array}$ \\
\hline 10 & 37.09 & $\begin{array}{c}40.59 \\
(+9.44)\end{array}$ & $\begin{array}{c}41.42 \\
(+11.67)\end{array}$ & $\begin{array}{c}40.16 \\
(+8.28)\end{array}$ \\
\hline 20 & 28.13 & $\begin{array}{c}30.43 \\
(+8.18)\end{array}$ & $\begin{array}{c}32.73 \\
(+16.35)\end{array}$ & $\begin{array}{c}34.33 \\
(+22.04)\end{array}$ \\
\hline 30 & 21.35 & $\begin{array}{c}24.84 \\
(+16.35)\end{array}$ & $\begin{array}{c}27.09 \\
(+26.89)\end{array}$ & $\begin{array}{c}29.69 \\
(+39.06)\end{array}$ \\
\hline 40 & 16.94 & $\begin{array}{c}21.19 \\
(+25.09)\end{array}$ & $\begin{array}{c}22.61 \\
(+33.47)\end{array}$ & $\begin{array}{c}23.54 \\
(+38.96)\end{array}$ \\
\hline 50 & 14.33 & $\begin{array}{c}17.60 \\
(+22.82)\end{array}$ & $\begin{array}{c}19.02 \\
(+32.73)\end{array}$ & $\begin{array}{c}21.00 \\
(+46.55)\end{array}$ \\
\hline 60 & 10.61 & $\begin{array}{c}13.33 \\
(+25.64)\end{array}$ & $\begin{array}{c}14.26 \\
(+34.40)\end{array}$ & $\begin{array}{c}15.90 \\
(+49.86)\end{array}$ \\
\hline 70 & 7.66 & $\begin{array}{c}9.87 \\
(+28.85)\end{array}$ & $\begin{array}{c}10.56 \\
(+37.86)\end{array}$ & $\begin{array}{c}11.66 \\
(+52.23)\end{array}$ \\
\hline 80 & 5.96 & $\begin{array}{c}6.65 \\
(+11.58)\end{array}$ & $\begin{array}{c}6.34 \\
(+6.38)\end{array}$ & $\begin{array}{c}8.59 \\
(+44.13)\end{array}$ \\
\hline 90 & 3.97 & $\begin{array}{c}3.85 \\
(-3.02)\end{array}$ & $\begin{array}{c}3.68 \\
(-0.73)\end{array}$ & $\begin{array}{c}4.98 \\
(+25.44)\end{array}$ \\
\hline 100 & 1.95 & $\begin{array}{c}0.96 \\
(-50.77)\end{array}$ & $\begin{array}{c}0.97 \\
(-50.26)\end{array}$ & $\begin{array}{c}1.99 \\
(+2.05)\end{array}$ \\
\hline Avg. & 16.55 & $\begin{array}{c}19.10 \\
(+15.41)\end{array}$ & $\begin{array}{c}19.67 \\
(+18.85)\end{array}$ & $\begin{array}{c}20.89 \\
(+26.77)\end{array}$ \\
\hline
\end{tabular}

\subsection{Case Studies}

In the above experiments, we observe that the VIPS algorithm successfully overcome, to some extents, the problems of noise and multi-topics.

We take query \#15 as an example to show how the impacts of noises can be eliminated by page segmentation. Query \#15 is "what about alexander graham bell?" that aims to retrieve relevant web pages about inventions by Alexander Graham Bell. The baseline precision of this query is $16.05 \%$. Table 3 lists the expansion terms and precisions of this query for different methods.

There are two strange words "iath" and "gorman" that are selected as expansion terms by FULLDOC. When we investigate the top 10 pages (two examples are shown in Figure 4), we find that the word "iath" appears on the bottom of the page "IATH WWW Server" and "gorman" is the author of the book "Alexander Graham Bell's Path to the Telephone", so his name appears on the bottom of the page as the copyright owner. Since these two words are not directly related to the original query, the final retrieval will get many unrelated documents containing these words, which decreases the search precision. 
Table 3. Expansion terms obtained for query \#15

\begin{tabular}{|c|c|c|c|}
\hline Methods & FULLDOC & DOMPS & VIPS \\
\hline $\begin{array}{c}\text { Expansion } \\
\text { Terms }\end{array}$ & $\begin{array}{l}\text { iath } \\
\text { notebooks } \\
\text { deaf } \\
\text { invention } \\
\text { gorman } \\
\text { simulating } \\
165 \\
1876 \\
\text { inventors } \\
\text { invent }\end{array}$ & $\begin{array}{l}\text { audiotelegraph } \\
1876 \\
\text { mehalik } \\
\text { oblon } \\
\text { prototype } \\
\text { industriously } \\
\text { technology } \\
\text { tinkerer } \\
\text { telephone } \\
\text { spattered }\end{array}$ & $\begin{array}{l}\text { invention } \\
\text { deaf } \\
1876 \\
\text { telephone } \\
\text { divestiture } \\
\text { edison } \\
\text { telephones } \\
\text { lineage } \\
\text { 1885 } \\
\text { inventors }\end{array}$ \\
\hline $\begin{array}{c}\text { Avg. } \\
\text { precision }\end{array}$ & $14.13 \%$ & $35.06 \%$ & $50.89 \%$ \\
\hline
\end{tabular}

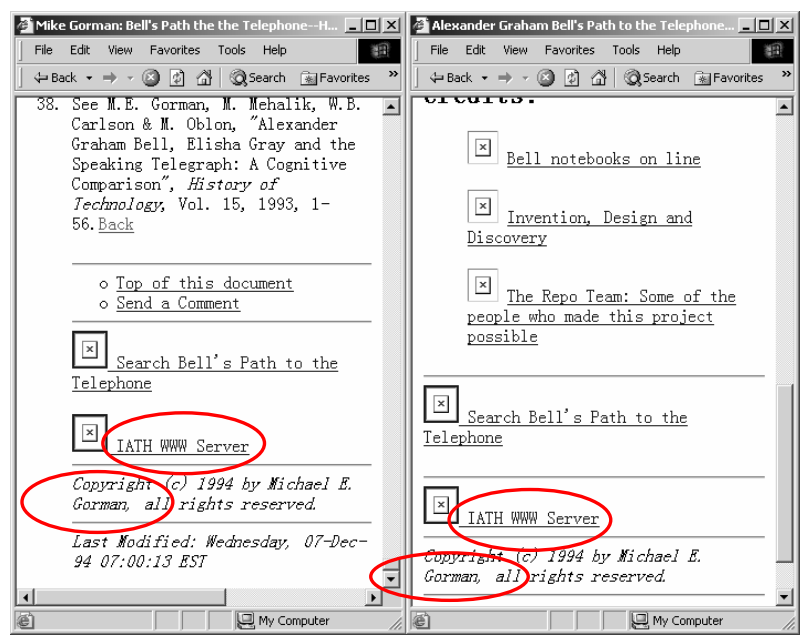

Figure 4. Example pages for query \#15 (DOCNO: WTX003-B35-115 and WTX004-B09-289)

To show how the multi-topics problem is dealt with by page segmentation, we choose query \#17 as an example. The query is "titanic what went wrong" and the baseline precision is $6.71 \%$. Within the top ranked web pages returned by the initial retrieval, all of the first, second and ninth documents are from the website "The Risks Digest" and have a similar structure as that in Figure 1. Two of them are shown in Figure 5. Because there are so many different topics in a single page, terms about the reasons leading to the sinking of the Titanic are hard to extract from the page. Many irrelevant words such as "call" and "maybe" in other topics are selected, which hurts the final retrieval performance. Table 4 shows the comparison of the selected expansion terms and precisions.

In summary, our experiments clearly show that page segmentation is an effective way to detect and filter out noisy and irrelevant information. Thus better expansion terms can be selected and the retrieval performance is improved. The quality of expansion terms is heavily dependent on the performance of page segmentation methods. VIPS produces more accurately web page segmentation at the semantic level and significantly outperform the simple DOMPS.

Table 4. Expansion terms obtained for query \#17

\begin{tabular}{|c|l|l|l|}
\hline Methods & FULLDOC & \multicolumn{1}{|c|}{ DOMPS } & \multicolumn{1}{c|}{ VIPS } \\
\hline & iceberg & britannic & sinking \\
& sri & sinking & unsinkable \\
& call & rms & iceberg \\
& maybe & iceberg & britannic \\
& actually & hmhs & rms \\
Expansion & noticed & passengers & passengers \\
& difference & tragedy & 1912 \\
& picked & ballard & ship \\
& theater & sank & cyberflix \\
& reasons & seawifs & ocean \\
\hline $\begin{array}{c}\text { Avg. } \\
\text { precision }\end{array}$ & $16.20 \%$ & $15.23 \%$ & $30.43 \%$ \\
\hline
\end{tabular}

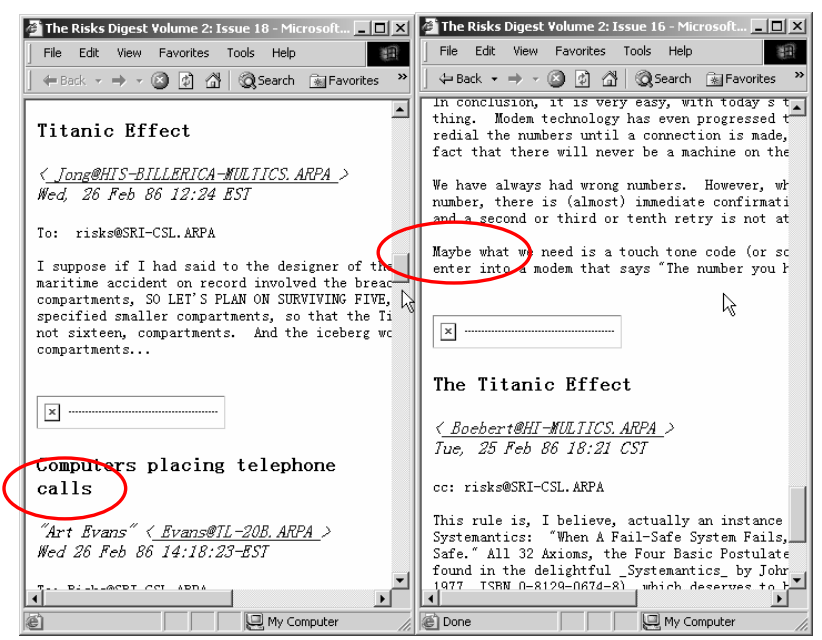

Figure 5. Example pages for query \#17

(DOCNO: WTX011-B22-202 and WTX011-B22-199)

\section{CONCLUSION}

Web information retrieval shows many differences compared to traditional text retrieval. Some traditional approaches which work well in traditional text retrieval, such as pseudo-relevance feedback, could not achieve the same level of performance in web information retrieval if applied directly.

We presented in this paper a novel web page segmentation algorithm called VIPS which constructs the content structure of a web page based on visual cues. This content structure better reflects the semantic structure of the web page. The algorithm is very efficient and scalable, and thus suitable for real time processing.

We applied VIPS to assist pseudo-relevance feedback in web information retrieval. The experimental results demonstrated that by partitioning a web page into semantically related units, better query expansion terms can be selected to improve the overall 
retrieval performance. We also compared our VIPS approach with DOM-based approach as well as full documents based approach. The results indicated that the best performance is achieved by our VIPS approach.

\section{REFERENCES}

[1] Baeza-Yates, R. and Ribeiro-Neto, B., Modern Information Retrieval, Chapter 13, Addison Wesley Longman 1999.

[2] Bailey, P., Craswell, N., and Hawking, D., Engineering a multi-purpose test collection for Web retrieval experiments, Information Processing and Management, in press.

[3] Buckley, C., Salton, G., and Allan, J., Automatic Retrieval with Locality Information Using Smart, In The First Text REtrieval Conference (TREC-1), National Institute of Standards and Technology, Gaithersburg, MD, 1992, pp. 5972 .

[4] Buttler, D., Liu, L., and Pu, C., A Fully Automated Object Extraction System for the World Wide Web, In International Conference on Distributed Computing Systems, 2001.

[5] Buyukkokten, O., Garcia-Molina, H., and Paepche, A., Accordion Summarization for End-Game Browsing on PDAs and Cellular Phones, In Proc. of the Conf. on Human Factors in Computing Systems, CHI'01, 2001.

[6] Chakrabarti, S., Integrating the Document Object Model with hyperlinks for enhanced topic distillation and information extraction, In the 10th International World Wide Web Conference, 2001.

[7] Chen, J., Zhou, B., Shi, J., Zhang, H., and Wu, Q., FunctionBased Object Model Towards Website Adaptation, In Proceedings of the 10th International World Wide Web Conference, 2001.

[8] Crivellari, F. and Melucci, M., Web Document Retrieval Using Passage Retrieval, Connectivity Information, and Automatic Link Weighting--TREC-9 Report, In The Ninth Text REtrieval Conference (TREC 9), 2000.
[9] Efthimiadis, N. E., Query Expansion, In Annual Review of Information Systems and Technology, Vol. 31, 1996, pp. 121-187.

[10] Embley, D. W., Jiang, Y., and Ng, Y.K., Record-boundary discovery in Web documents, In Proceedings of the 1999 ACM SIGMOD international conference on Management of data, Philadelphia PA, 1999, pp. 467-478.

[11] Gu, X., Chen, J., Ma, W.Y., and Chen, G., Visual Based Content Understanding towards Web Adaptation, In Second International Conference on Adaptive Hypermedia and Adaptive Web-based Systems (AH2002), Spain, 2002, pp. 29-31.

[12] Kaasinen, E., Aaltonen, M., Kolari, J., Melakoski, S., and Laakko, T., Two Approaches to Bringing Internet Services to WAP Devices, In Proceedings of 9th International WorldWide Web Conference, 2000, pp. 231-246.

[13] Newby, G., Information Space Based on HTML Structure, In The Ninth Text REtrieval Conference (TREC 9), 2000, pp. 601-610.

[14] Robertson, S. E., Overview of the okapi projects, Journal of Documentation, Vol. 53, No. 1, 1997, pp. 3-7.

[15] Robertson, S. E. and Sparck Jones, K., Relevance weighting of search terms, Journal of the American Society of Information Science, Vol. 27, No. May-June, 1976, pp. 129146.

[16] Robertson, S. E. and Walker, S., Okapi/Keenbow at TREC-8, In The Eighth Text REtrieval Conference (TREC 8), 1999, pp. 151-162.

[17] Yang, Y. and Zhang, H., HTML Page Analysis Based on Visual Cues, In 6th International Conference on Document Analysis and Recognition (ICDAR 2001), Seattle, Washington, USA, 2001. 